\title{
Luciferase and Bioluminescence Microscopy for Analyses of Membrane Dynamics in Living Cells
}

\section{Naohiro Kato*}

Associate Professor, Department of Biological Sciences, Louisiana State University, USA

Luciferase-based bioluminescence is a technique used for non-invasive analysis of molecular functions in living cells. New recombinant luciferase and advanced CCD (Charge Coupled Device) cameras allow the detection of bioluminescent signals at submicron resolution. These recombinant luciferase proteins have the potential to facilitate great advances in the study of membrane dynamics at both the subcellular and whole organism levels.

Bioluminescence is the emission of light generated by the enzymatic catalysis of a chemical substrate. This is different than fluorescence which is the emission of light generated by the absorption of photons. While the brightness of a fluorescent body correlates with its molar extinct coefficient (ability of a molecule to absorb photons) and quantum yield (ability of a molecule to emit photons), brightness of bioluminescent body correlates with a concentration of the substrate and efficiency of the bioluminescent enzyme in catalyzing the substrate.

Fluorescence microscopy is widely used to analyse functions of molecules in living cells. Genetically engineered fluorescent proteins that are fused to a protein of interest via recombinant DNA techniques can determine subcellular localization of a protein of interest. From a membrane science and technology point of view, recombinant fluorescent proteins have been used to study membrane-associated processes in living cells such as endocytosis [1], morphological changes of the Golgi complex [2], and protein clusters in the membrane [3]. It is difficult to use fluorescent proteins for in vivo analyses because it is difficult for the excitation light to reach a fluorescent protein located in cells buried deep within a tissue or organism. Moreover, background fluorescence is relatively high because many tissues contain molecules that naturally fluorescence. Also, the working distance of most compound microscope objectives is very small (millimeters). Therefore, the use of fluorescent proteins in living cells is restricted to in vitro experiments except in special cases $[4,5]$.

Luciferase-based bioluminescence, on the other hand, has been widely used for various experiments in whole organisms. A luciferase substrate is small and permeable to the plasma membrane, so that it is readily delivered to target cells even when these cells are located inside of a tissue or an intact organism. Moreover, because many cells do not naturally generate light, bioluminescence assays do not suffer from the background noise which complicates similar analyses using fluorescence. A drawback of using traditional luciferase-based bioluminescence is that the total number of photons emitted is normally too low to detect with a conventional microscope and CCD camera. This fact makes microscopic detection of bioluminescence a challenge in vitro [6].

Advances in both microscopy and the luciferase gene itself are removing these challenges. Until recently, luciferase from firefly (Lampyridae) was the only commercially available luciferase for the bioluminescence assay. Recently luciferase from different families has been isolated and commercialized. The commercialized luciferase genes include the click beetle (Elateridae), sea pansy (Renillidae), and marine copepod (Metridinidae) luciferase. The beetle luciferase provides two homologous clones that emit green $(544 \mathrm{~nm})$ and red
(611 nm) light (Promega, WI), which allows one to simultaneously monitor two biological activities in a cell. Sea pansy (Renilla luciferase, Promega) and marine copepod (Gaussia luciferase, Marker Gene Technologies, OR) luciferase provide brighter bioluminescence signals than firefly luciferase. Especially, Gaussia luciferase is reported to emit 100 fold brighter signals than firefly luciferasein living cells [7]. The smaller molecular sizes of Renilla (36k Da) and Gaussia (20k Da) luciferase as compared to that of firefly luciferase $(61 \mathrm{k} \mathrm{Da})$ provide an additional benefit to those trying to avoid size constraints inherent in some biological systems. The substrate of Renilla and Gaussia luciferase is coelenterazine, which is structurally different from luciferin, the substrate of firefly and click beetle luciferase. Unlike firefly luciferase that emits green light $(\sim 560 \mathrm{~nm})$ during the enzymatic catalysis of luciferin, Renilla and Gaussia luciferase emits cyan light $(\sim 480 \mathrm{~nm})$ during the enzymatic catalysis of coelenterazine.

One potential problem with Renilla and Gaussia luciferaces lies in this cyan light emission. The cyan light often scatters in tissues of an analysed organism due to its short wavelength. This makes the number of photons reaching the detector low during in vivo experiments. To counter this problem a recombinant Renilla luciferase was developed that makes the catalysing coelentazaline emit green light $(535 \mathrm{~nm})$. The recombinant Renilla luciferase is calculated to transmit light more than 10 fold in vivo as compared to the originally cloned Renilla luciferase $[8,9]$.

Another technological development to aid in the detection of bioluminescence in living cells is bioluminescence microscopy (Olympus, Japan; ATTO, Japan) which uses simplified optics to reduce the loss of photons and an EM (Electron Multiplying)-CCD camera which amplifies electron signals received by each sensor while minimizing the read-noise. Quantum efficiency (ability to convert light to electron signals) of EM-CCD is drastically improved (over $90 \%$ for $500-600 \mathrm{~nm}$ light), compared to that of conventional CCDs $(\sim 50 \%$ for $500-600 \mathrm{~nm}$ light). Furthermore, the microscope is enclosed in a dark box to ensure that no external light is in the system. As the result of the technological implementations, the microscopy allows taking bioluminescence image with short exposure time at a submicron resolution [10-12].

Further modifications to both the luciferase protein and its placement within the cell indicate even more versatility for this system.

*Corresponding author: Naohiro Kato, 226 Life Sciences Building, Louisiana State University, Baton Rouge, LA70808, USA, Tel: 225-578-2004; Fax: 225-5782597; E-mail: kato@Isu.edu

Received July 18, 2012; Accepted July 20, 2012; Published July 23, 2012

Citation: Kato N (2012) Luciferase and Bioluminescence Microscopy for Analyses of Membrane Dynamics in Living Cells. J Memb Sci Technol 2:e109. doi:10.4172/2155-9589.1000e109

Copyright: (c) 2012 Kato N. This is an open-access article distributed under the terms of the Creative Commons Attribution License, which permits unrestricted use, distribution, and reproduction in any medium, provided the original author and source are credited. 
Citation: Kato N (2012) Luciferase and Bioluminescence Microscopy for Analyses of Membrane Dynamics in Living Cells. J Memb Sci Technol 2:e109. doi:10.4172/2155-9589.1000e109

Page 2 of 2

A Gaussia luciferase protein targeted to the plasma membrane was used to monitor luciferase-tagged $\mathrm{T}$ cells accumulating in a tumor in a whole organism noninvasively [13]. These investigators found that the luciferase signal was enhanced by localizing the luciferase to the plasma membrane rather than inside the cell which allowed them to detect the small groups of cells. Another recently developed use for luciferase, called the luciferase complementation assay, was used to detect a hormone-dependent protein interaction in living cells [14]. The luciferase complementation assay utilizes a luciferase that has been split into $\mathrm{N}$ - and C-terminal domains. These domains are not enzymatically active when expressed alone or together; however if these domains are genetically fused to proteins, via recombinant DNA techniques, which interact with each other, their enzymatic activity is restored. The complementation assay has been used to discover a membrane-protein complex [15,16], mitochondria fusion [17], and SNARE interactions that are involved in vesicle fusion in living cells [18]. Split luciferase complementation assays using Renilla and Guasian luciferase also have been developed [6,19].

In the very near future, combining the new recombinant luciferase and microscopy techniques described here could reveal living cell membrane dynamics in a transgenic organism at various scales, from subcellular to whole organism, in both in vitro and in vivo settings. New luciferase clones have been commercialized in many countries, while bioluminescence microscopy has been commercialized in Europe and Japan only. It is going to be available in the US later in 2012

\section{References}

1. Gaidarov I, Santini F, Warren RA, Keen JH (1999) Spatial control of coated-pit dynamics in living cells. Nat Cell Biol 1: 1-7.

2. Sciaky N, Presley J, Smith C, Zaal KJ, Cole N, et al. (1997) Golgi tubule traffic and the effects of brefeldin A visualized in living cells. J Cell Biol 139: 11371155.

3. Sieber JJ, Willig KI, Kutzner C, Gerding-Reimers C, Harke B, et al. (2007) Anatomy and dynamics of a supramolecular membrane protein cluster. Science 317: 1072-1076.

4. Junt T, Schulze H, Chen Z, Massberg S, Goerge T, et al. (2007) Dynamic visualization of thrombopoiesis within bone marrow. Science 317 : 1767-1770.

5. Levene MJ, Dombeck DA, Kasischke KA, Molloy RP, Webb WW (2004) In vivo multiphoton microscopy of deep brain tissue. J Neurophysiol 91: 1908-1912.

6. Kaihara A, Kawai Y, Sato M, Ozawa T, Umezawa Y (2003) Locating a proteinprotein interaction in living cells via split Renilla luciferase complementation. Anal Chem 75: 4176-4181.

7. Tannous BA, Kim DE, Fernandez JL, Weissleder R, Breakefield XO (2005) Codon-optimized Gaussia luciferase cDNA for mammalian gene expression in culture and in vivo. Mol Ther 11: 435-443.

8. Loening AM, Wu AM, Gambhir SS (2007) Red-shifted Renilla reniformis luciferase variants for imaging in living subjects. Nat Methods 4: 641-643.

9. Loening AM, Fenn TD, Wu AM, Gambhir SS (2006) Consensus guided mutagenesis of Renilla luciferase yields enhanced stability and light output Protein Eng Des Sel 19: 391-400.

10. Akashi M, Hayasaka N, Yamazaki S, Node K (2008) Mitogen-activated protein kinase is a functional component of the autonomous circadian system in the suprachiasmatic nucleus. J Neurosci 28: 4619-4623.

11. Yagita K, Horie K, Koinuma S, Nakamura W, Yamanaka I, et al. (2010) Development of the circadian oscillator during differentiation of mouse embryonic stem cells in vitro. Proc Natl Acad Sci U S A 107: 3846-3851.

12. Binkowski B, Fan F, Wood K (2009) Engineered luciferases for molecular sensing in living cells. Curr Opin Biotechnol 20: 14-18.

13. Santos EB, Yeh R, Lee J, Nikhamin Y, Punzalan B, et al. (2009) Sensitive in vivo imaging of $T$ cells using a membrane-bound Gaussia princeps luciferase. Nat Med 15: 338-344.

14. Ozawa T, Kaihara A, Sato M, Tachihara K, Umezawa Y (2001) Split luciferase as an optical probe for detecting protein-protein interactions in mammalian cells based on protein splicing. Anal Chem 73: 2516-2521.

15. Luker KE, Gupta M, Luker GD (2008) Imaging CXCR4 signaling with firefly luciferase complementation. Anal Chem 80: 5565-5573.

16. Dagda RK, Barwacz CA, Cribbs JT, Strack S (2005) Unfolding-resistant translocase targeting: a novel mechanism for outer mitochondrial membrane localization exemplified by the Bbeta2 regulatory subunit of protein phosphatase 2A. J Biol Chem 280: 27375-27382.

17. Huang $\mathrm{H}$, Choi SY, Frohman MA (2010) A quantitative assay for mitochondria fusion using Renilla luciferase complementation. Mitochondrion 10: 559-566.

18. Kato N, Fujikawa Y, Fuselier T, Adamou-Dodo R, Sato MH, et al. (2009) Luminescence detection of SNARE-SNARE interaction in Arabidopsis protoplasts. Plant Mol Biol 72: 433-444.

19. Remy I, Michnick SW (2006) A highly sensitive protein-protein interaction assay based on Gaussia luciferase. Nat Methods 3: 977-979. 